\title{
A high activity index of stearoyl-CoA desaturase is associated with increased risk of fracture in men
}

\author{
H. Melhus • U. Risérus • E. Warensjö • L. Wernroth • \\ K. Jensevik $\cdot$ L. Berglund $\cdot$ B. Vessby $\cdot$ K. Michaëlsson
}

Received: 28 August 2007 / Accepted: 5 November 2007 / Published online: 8 December 2007

(C) The Author(s) 2007

\begin{abstract}
Summary The activity index of stearoyl-CoA desaturase (SCD), a key enzyme in lipogenesis, was associated with increased risk of fracture in a longitudinal population-based cohort of men. This indicates that elevated levels of endogenous lipogenesis increase the risk of fracture and suggest a role for saturated fat in the pathogenesis of osteoporosis.

Introduction Osteoblasts and marrow adipocytes are derived from a common mesenchymal progenitor, and experimental studies have indicated that increased adipogenesis can occur at the expense of osteoblasts, leading to bone loss. Stearoyl-CoA desaturase (SCD) converts saturated to monounsaturated fatty acids and is a key enzyme in lipogenesis.

Methods Analysis was performed in a population-based, longitudinal cohort study of men $(n=2009)$. A product-toprecursor index (palmitoleic acid/palmitic acid) was used to
\end{abstract}

\footnotetext{
H. Melhus $(\bowtie)$

Department of Medical Sciences, University Hospital,

Entrance 61, 4th floor,

SE-751 85 Uppsala, Sweden

e-mail: Hakan.Melhus@medsci.uu.se

U. Risérus $\cdot$ E. Warensjö • B. Vessby

Clinical Nutrition and Metabolism, Department of Public Health and Caring Sciences, University Hospital,

Uppsala, Sweden

L. Wernroth $\cdot$ K. Jensevik $\cdot$ L. Berglund $\cdot K$. Michaëlsson Uppsala Clinical Research Center, University Hospital, Uppsala, Sweden

K. Michaëlsson

Department of Surgical Sciences, University Hospital,

Uppsala, Sweden
}

estimate SCD activity in fasting serum analyzed in samples obtained at enrollment at age 50 years. Fractures were documented in 422 men during 35 years of follow-up. Cox regression analysis was used to determine the risk of fracture according to SCD activity index.

Results The risk of fracture was highest among men with the highest levels of SCD activity index. Multivariable analysis of the risk of fracture in the highest quintile as compared to the lowest one showed that the rate ratio was 1.71 (95\% CI 1.26-2.33) for any fracture, with an estimated population attributable risk of $15 \%$. The risk was further increased within the highest quintile.

Conclusions Our results indicate that elevated levels of endogenous lipogenesis increase the risk of fracture and suggest a role for saturated fat in the pathogenesis of osteoporosis.

Keywords Fat · Fracture - Lipogenesis · Men · Nutrition . Osteoporosis

\section{Introduction}

In the last few years, it has become apparent that there is a reciprocal relationship between the development of bone and fat. Osteoblasts and marrow adipocytes share a common mesenchymal progenitor and bone loss is associated with an expansion of adipose tissue in the marrow [1]. Recent animal studies have shown that the peroxisome proliferator-activated receptor gamma (PPAR $\gamma$ ) pathway, i.e., the dominant regulator of adipogenesis, inhibits osteoblast differentiation [2], and that leptin, an anorexigenic metabolic hormone that is secreted in proportion to fat mass, is a regulator of both bone formation and resorption [3-5]. 
We hypothesized that a key enzyme in lipogenesis, stearoyl-CoA desaturase (SCD) may be of particular interest in this context. SCD is involved in the cellular biosynthesis of monounsaturated fatty acids (MUFAs) from saturated fatty acid (SFA) substrates (mainly palmitic and stearic acid). As a result of substrate channeling the major MUFAs generated by SCD, i.e., palmitoleic and oleic acid, represent the main substrates for synthesis of triglycerides, cholesterol esters and phospholipids. The palmitoleic/ palmitic acid (16:1/16:0) index in serum, also known as the "desaturation index" has been used to estimated SCD activity $[6,7]$. Consistent with a role as a key point of metabolic control, SCD activity is tightly regulated, being decreased by polyunsaturated fatty acids (PUFAs) and increased by SFAs [8]. Interestingly, SCD-1-null mice display resistance to obesity and hyperlipidemia [9]. The levels of palmitoleic acid have been shown to be significantly associated with abdominal adiposity in obese female twins [10] and in obese children [11]. We have recently shown that the SCD activity index is significantly associated with obesity in both men and women [12] and that PPAR $\gamma$ function appears to have a pivotal role in SCD regulation [7]. This is the first study to investigate the relation between the SCD activity and risk of fracture.

\section{Methods}

\section{Subjects}

The Uppsala Longitudinal Study of Adult Men (ULSAM) has been described previously [13, 14]. Briefly, from 1970 to 1973 , we invited all 2,841 men, born 1920-1924, living in the municipality of Uppsala, Sweden, to participate in a health survey, the Uppsala Longitudinal Study of Adult Men. A total of 2,322 men ( $82 \%$ of those invited), 49 to 51 years of age, agreed to participate. At 60 years of age 1,860 men took part in a second evaluation, at 70 years 1,221 men took part in a third evaluation, and at 77 years 839 men participated and finally 530 participated in the final evaluation at age 82 years. At end of follow-up (Dec $31,2005), 1295$ (56\% of the cohort) of these men had died.

\section{SCD activity index}

Fasting serum palmitoleic acid and palmitic acid in ULSAM were analyzed in cholesterol esters by gas-liquid chromatography after separation of serum lipids by thinlayer chromatography as previously described [15-17]. Briefly, serum was extracted with a hexane-isopropanol solution and cholesteryl esters (only lipid esters that were measured at baseline) were separated from the extract by thin-layer chromatography before inter-esterification with acidic methanol was performed. Free cholesterol that had been liberated in the reaction was removed by aluminium oxide to avoid contamination of the column. The composition of methylated fatty acids was determined by gas chromatography (25 m NB-351 silica capillary column) with a flame ionization detector and helium as carrier gas. Every 25th sample was a serum control pool. The CV between successive gas chromatography runs was $0.2-5 \%$. A product-to-precursor index (palmitoleic acid 16:1 n-7/ palmitic acid 16:0) was used to estimate SCD activity index for the 2,009 men for which data were available at baseline.

\section{Identification of cases of fractures}

We sought to identify all fractures that occurred among the study participants after enrollment. We matched the study cohort to the Hospital Discharge Register to identify cases treated on an inpatient basis. All orthopaedic records, radiographic records and outpatient registries at the local hospitals in areas where the participants in the initial investigation resided were reviewed to identify out-patient treated fractures. We censored six cases of fracture caused by metastatic cancer. Seven cases of fractures due to suspected high-impact trauma were retained in the analysis since there are indications of comparable increases of low and high trauma fracture risk with decreasing bone density in elderly.

\section{Statistical analysis}

We used Cox proportional-hazards models to estimates rate ratios, with $95 \%$ confidence intervals calculated as measures of associations in the same way as previously described for serum retinol in the ULSAM cohort [13]. For each man, the number of years of follow-up was calculated from the date of enrollment (i.e., the date of the first investigation) until the date of a first fracture or the end of the follow-up period (December 31, 2005). Dates of deaths were based on data from the continuously updated Swedish National Population Register.

SCD activity index levels at baseline at 50 years of age were evaluated both as a continuous variable and as a categorical variable, in quintiles. Separate analyses were performed for fractures specifically designated as osteoporotic (i.e., fractures of the hip, pelvis, spine, distal forearm, and proximal humerus). The results were similar whether or not we included the seven cases of fractures due to suspected high-impact trauma, and these cases were therefore retained in the analyses. We then estimated the trend in the risk of fracture by restricted cubic-spline Cox regression with five "knots" (SCD activity index percentiles $5,27.5,50,72.5$, and 95), which enabled us to investigate extreme SCD values. We used the midpoint of the first 
quintile, i.e., percentile 10 , as reference point in this analysis. The results of this analysis are presented as smoothed plots with $95 \%$ CI for the overall risk of fracture.

In addition, since fatty acids were analyzed in half of the men participating in the third evaluation at 70 years $(n=611)$, we could examine the change of SCD activity index from age 50 to 70 years and the risk of fracture (in tertiles). Baseline in this particular analysis was age at the third investigation and fractures among these 611 men after this investigation were used as outcome $(n=66)$.

We considered a crude and a multivariable model. The latter included age, weight, height, serum retinol (all continuous), smoking (never, former, current), diabetes, (yes, no), marital status (single, divorced/widowed, married/ cohabitee), any cardiovascular disease (yes, no), self-rated health (good, not good), and physical activity (low, medium, high). The estimates remained similar when we also included sedimentation rate (at age 50), as well as alcohol intake, dietary energy intake and use of oral calcium and vitamin D (all at the age of 70 years). Consequently, these variables were omitted from the reported analyses.

The population attributable risk for fracture among those with a specific SCD-1 level was calculated by

$\frac{\mathrm{p}(\mathrm{RR}-1)}{[\mathrm{p}(\mathrm{RR}-1)+1]}$

where $p$ is the prevalence of the investigated SCD level among the fracture cases.

\section{Results}

Characteristics of the participants according to the quintile of SCD-1 activity index at baseline are shown in Table 1. There was a tendency toward higher weight, higher serum retinol values, more current smokers, more singles and divorced men, more circulatory system disease, lower selfrated health, and lower physical activity in higher quintiles of SCD-1 activity index. During 51,707 person-years of follow-up, we identified 422 men (21\%) with any fracture after study entry.

The overall risk of fracture increased by $31 \%$ for every increase of $1 \mathrm{SD}$ in SCD activity index. The increment was mainly concentrated in the highest quintile for SCD; multivariable rate ratio for any fracture, as compared with the lowest quintile, 1.71 (95\% CI 1.26-2.33) (Table 2), with an estimated population attributable risk of $15 \%$. For fractures at sites that are typical of osteoporotic fractures, the multivariable rate ratio was 1.91 (95\% CI 1.26-2.91). There was also a trend towards increased risk of hip fractures when analyzed as a continuous variable $(p=0.04)$, but the multivariable rate ratio for the highest quintile as compared with the lowest quintile did not reach statistical significance, HR 1.4 (95\% CI 0.8-2.6).

The risk of fracture was further increased in the highest quintile for SCD. Subjects with SCD levels in the 95th percentile had an overall risk of fracture that was about twice the risk among those with lower levels (crude rate ratio 2.8 (95\% CI 2.0-4.0), multivariable rate ratio 2.2

Table 1 Base-line characteristics of the study participants according to the quintile of SCD activity index levels (SD in parentheses)

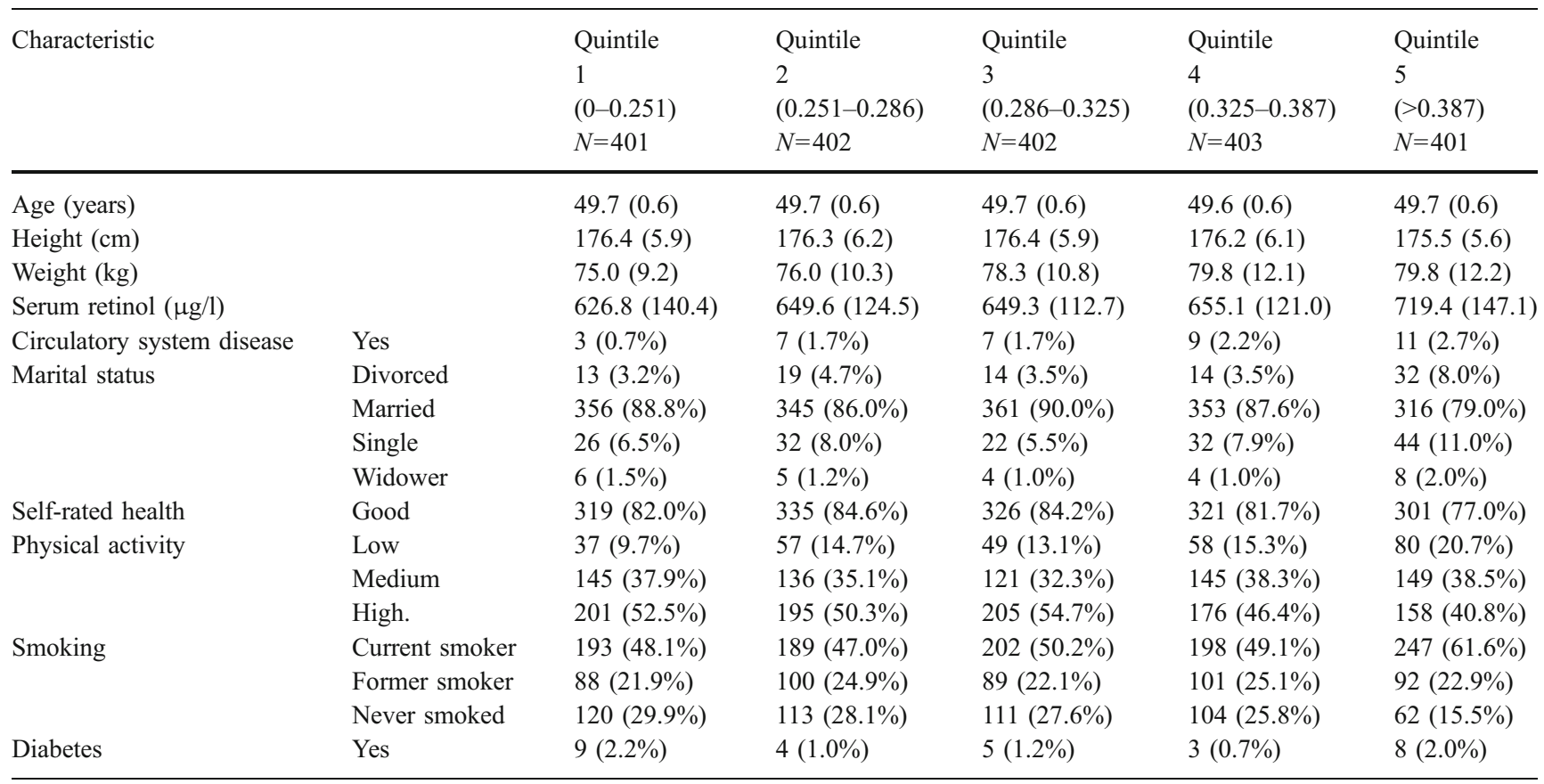


Table 2 Rate ratios (RRs) with 95\% confidence intervals (95\% CI) of any fracture by SCD activity index at baseline at 50 years

\begin{tabular}{|c|c|c|c|c|c|}
\hline \multirow[t]{2}{*}{ Category } & \multirow[t]{2}{*}{$n$ fractures } & \multicolumn{2}{|c|}{ Univariate analysis } & \multicolumn{2}{|c|}{$\begin{array}{l}\text { Multivariable* } \\
\text { analysis }\end{array}$} \\
\hline & & $\mathrm{RR}$ & $95 \% \mathrm{CI}$ & $\mathrm{RR}$ & $95 \% \mathrm{CI}$ \\
\hline Quintile 1 & 83 & 1.0 & Ref & 1.0 & Ref \\
\hline Quintile 2 & 84 & 1.03 & $0.76-1.40$ & 1.04 & $0.77-1.41$ \\
\hline Quintile 3 & 77 & 1.05 & $0.77-1.43$ & 1.09 & $0.80-1.50$ \\
\hline Quintile 4 & 73 & 1.08 & $0.79-1.48$ & 1.07 & $0.77-1.47$ \\
\hline Quintile 5 & 105 & 1.91 & $1.43-2.55$ & 1.71 & $1.26-2.33$ \\
\hline Per 1 SD & & 1.38 & $1.26-1.50$ & 1.31 & $1.19-1.44$ \\
\hline$P$ value & & $<0.001$ & & $<0.0$ & \\
\hline
\end{tabular}

* Adjusted for age, height, weight, serum retinol (all continuous), smoking status (never, former, current smoker), diabetes (yes/no), marital status (single, divorced/widowed, married/cohabiting), circulatory system disease (yes/no), self-rated health (good, not good), and physical activity (low, medium, high).

(95\% CI 1.5-3.2), whereas subjects in the 99th percentile had an overall risk that exceeded the risk among men with levels by a factor of about five (crude rate ratio 6.1 (95\% CI $3.3-11.5)$, multivariable rate ratio $4.52(95 \%$ CI $2.33-8.75)$ (Fig. 1).

In half of the men examined at 70 years of age, serum palmitoleic and palmitic acid were measured $(n=611)$. Using only fracture data that occurred in this sub-group (66 fractures), we found a significant correlation between the change in SCD index and fracture risk (crude rate ratio for third vs. lowest tertile 3.23 (95\% CI 1.50-6.93), multivariable rate ratio 2.16 (95\% CI 1.17-3.98), indicating that when the SCD activity index increases over time, so does the risk of fracture. In 454 men waist circumference was measured at enrollment. There was a moderate, but highly significant, correlation between SCD activity index and waist circumference $(\mathrm{r}=0.22, p<0.001)$.

\section{Discussion}

SCD is a key enzyme in the biosynthesis of MUFAs from SFAs, i.e., in lipogenesis. In this prospective, populationbased cohort study of men, the overall risk of fracture was substantially increased among the men with high levels of estimated SCD activity. The risk was concentrated in the highest quintile, with a continuous increase within this category, and it was independent of BMI. Thus, despite a positive effect of increased fat mass on weight-bearing, our results indicate that increased endogenous lipogenesis has negative effects on bone. Although we could only analyze a subgroup, the finding that an increase in SCD activity index from 50 to 70 years is associated with an even higher risk, gives further support to this.
We included not only height and weight in our multivariable model, but also a number of lifestyle factors. Adjustment for these factors had only modest effects on the estimates, indicating that the association between SCD activity index and fracture risk cannot be explained by these potential confounders. Instead our results suggest that the dietary fat quality and endogenous fatty acid metabolism may be important. It should be emphasized that increased SCD activity index does not directly reflect increased proportions of palmitoleic acid in the diet, since the content of this fatty acid is normally small in the diet. Rather, it reflects increased endogenous desaturation of palmitic acid by SCD leading to increased palmitoleic acid, as a consequence of a high intake of palmitic acid in the diet [11]. Interestingly, in glycogen storage disease type 1 , the synthesis of palmitic acid is increased 40-fold [18] and osteopenia is a known complication in this disease [19-22]. Furthermore, in animal studies, inclusion of palmitic acid as the major source of fat causes a reduction in bone ash and bone calcium levels [23, 24].

Alternatively, although unlikely in a Western diet with a high dietary intake of fat [25], an increased SCD activity index could be a consequence of a high intake of carbohydrates. At low fat intakes, the amount of palmitoleic acid in serum has been reported to reflect the hepatic pool of carbon flux from carbohydrates to fatty acids [26], e.g., in rats rendered obese by sucrose administration, SCD activity index increases dramatically in liver and plasma [27].

SFAs, high carbohydrate diets, and insulin all appear to be able to induce SCD expression, whereas dietary PUFAs and leptin seem to inhibit SCD mRNA transcription [11]. Therefore, the relationship between SCD and risk of fracture may reflect compensatory up-regulation of SCD to continuous intake of e.g., a diet high in SFAs (such as dairy products, solid margarines, and meat products) and low in PUFAs, rather than a metabolically harmful effect of increased activity in itself [17]. Consistent with this, a

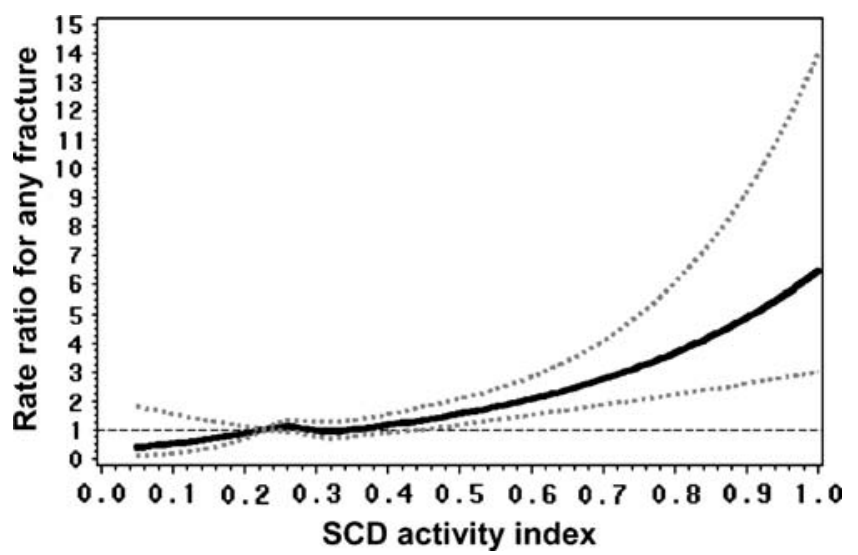

Fig. 1 Smoothed plot of rate ratios for any fracture according to the adjusted SCD activity index level at baseline 
recent analysis of NHANES III by Corwin et al. [28] found that SFAs are inversely associated with bone density at several hip sites, particularly in men $<50$ years of age.

SCD activity can also be estimated as the oleic/stearic acid (18:1/18:0) ratio [6]. However, the predictive value of this ratio in serum in the ULSAM cohort has previously been shown to be considerably less than for palmitoleic/ palmitic acid due to a much higher dietary proportion of oleic acid [17], and was therefore not analyzed in this study.

Mounting evidence indicates an important role of PPAR $\gamma$ in skeletal metabolism. PPAR $\gamma$ haploinsufficient mice have increased bone mass and decreased adipogenesis, whereas activation of PPAR $\gamma$ with the agonist rosiglitazone causes bone loss and increased bone marrow adiposity in mice [29, 30]. Thiazolidinedione use has recently been shown to cause bone loss also in humans [31]. In a randomized clinical trial rosiglitazone increased SCD activity index, and in one patient with partial lipodystrophy and type 2 diabetes due to dominant-negative mutation in the PPAR $\gamma$ gene, it restored SCD activity index, indicating a pivotal role of PPAR $\gamma$ function in SCD regulation, at least in adipose tissue [7]. The activation of PPAR $\gamma$ by natural ligands, such as oxidized fatty acids, plays an important role in the development of atherosclerosis [32]. Oxidized lipids also inhibit osteoblast development in vitro and bone formation in vivo, and serve as substrates for PPAR $\gamma$, which redirects differentiation of mesenchymal cells from osteoblasts to adipocytes. PPAR also enhances many adipocyte genes and diminishes Cbfal/ Runx2, needed for osteoblast development. All these effects could reduce bone formation and promote atherogenesis [33]. Atherosclerosis could be one of the common factors which would explain the reported relationship between cardiovascular disease and osteoporosis [34]. Our findings are consistent with previous findings from the ULSAM cohort regarding serum fatty acid profile and the risk of developing cardiovascular disease [16, 35]. Moreover, inflammation plays an important role in atherogenesis. Part of the inflammatory response is activation of $\mathrm{T}$ cells with release of cytokines, especially of RANKL, which is a key molecule in bone resorption [33].

We have previously found an association between serum retinol and fracture risk in ULSAM (12). Retinol is a fatsoluble vitamin found in dietary fat from animal sources, i.e., in food that may also contain high levels of SFAs. However, inclusion of retinol in the multivariable model had only minor effects on the rate ratio.

Our population-based study had the advantage of involving men with similar age followed from a time before they reached the high incidence ages for fractures, and we used hospital-record verification of fractures. In addition, we used serum levels of individual fatty acids, rather than dietary assessments of fat intake.
There are some limitations of this study. The study population consisted only of men and therefore our results need to be verified in women. Since direct measures of SCD activity is very difficult to obtain in larger studies, we used the ratio between two fatty acids. Whether the ratio mainly reflects SCD activity in adipose tissue, liver, or both still remains to be elucidated. Unfortunately, bone mineral density data were not available. Another limitation of the study is the lack of dietary records at baseline and the resulting lack of knowledge about the intake of e.g., SFAs and PUFAs in the studied population.

In conclusion, the results of our study suggest that a high SCD activity, indicating elevated endogenous lipogenesis, substantially increases the risk of fractures. Previous experimental work showing a reciprocal relationship between bone and fat supports this conclusion.

Acknowledgements This study was supported by the Swedish Research Council (projects no 12671 and 13511), Torsten and Ragnar Söderberg Foundations, and the Swedish Society for Medical Research.

We would like to acknowledge our sincere gratitude to the late professor Hans Lithell $(† 2005)$ for his efforts to preserve the ULSAM cohort. This study would not have been possible to perform without his work.

\section{Conflicts of interest None.}

Open Access This article is distributed under the terms of the Creative Commons Attribution Noncommercial License which permits any noncommercial use, distribution, and reproduction in any medium, provided the original author(s) and source are credited.

\section{References}

1. Parhami F, Morrow AD, Balucan J, Leitinger N, Watson AD, Tintut Y, Berliner JA, Demer LL (1997) Lipid oxidation products have opposite effects on calcifying vascular cell and bone cell differentiation. A possible explanation for the paradox of arterial calcification in osteoporotic patients. Arterioscler Thromb Vasc Biol 17:680-687

2. Akune T, Ohba S, Kamekura S, Yamaguchi M, Chung UI, Kubota N, Terauchi Y, Harada Y, Azuma Y, Nakamura K, Kadowaki T, Kawaguchi H (2004) PPARgamma insufficiency enhances osteogenesis through osteoblast formation from bone marrow progenitors. J Clin Invest 113:846-855

3. Takeda S, Elefteriou F, Levasseur R, Liu X, Zhao L, Parker KL, Armstrong D, Ducy P, Karsenty G (2002) Leptin regulates bone formation via the sympathetic nervous system. Cell 111:305-317

4. Elefteriou F, Takeda S, Ebihara K, Magre J, Patano N, Kim CA, Ogawa Y, Liu X, Ware SM, Craigen WJ, Robert JJ, Vinson C, Nakao K, Capeau J, Karsenty G (2004) Serum leptin level is a regulator of bone mass. Proc Natl Acad Sci USA 101:3258-3263

5. Elefteriou F, Ahn JD, Takeda S, Starbuck M, Yang X, Liu X, Kondo H, Richards WG, Bannon TW, Noda M, Clement K, Vaisse C, Karsenty G (2005) Leptin regulation of bone resorption by the sympathetic nervous system and CART. Nature 434:514-520

6. Attie AD, Krauss RM, Gray-Keller MP, Brownlie A, Miyazaki M, Kastelein JJ, Lusis AJ, Stalenhoef AF, Stoehr JP, Hayden MR, Ntambi JM (2002) Relationship between stearoyl-CoA desaturase 
activity and plasma triglycerides in human and mouse hypertriglyceridemia. J Lipid Res 43:1899-1907

7. Riserus U, Tan GD, Fielding BA, Neville MJ, Currie J, Savage DB, Chatterjee VK, Frayn KN, O'Rahilly S, Karpe F (2005) Rosiglitazone increases indexes of stearoyl-CoA desaturase activity in humans: link to insulin sensitization and the role of dominant-negative mutation in peroxisome proliferator-activated receptor-gamma. Diabetes 54:1379-1384

8. Liew CF, Groves CJ, Wiltshire S, Zeggini E, Frayling TM, Owen KR, Walker M, Hitman GA, Levy JC, O'Rahilly S, Hattersley AT, Johnston DG, McCarthy MI (2004) Analysis of the contribution to type 2 diabetes susceptibility of sequence variation in the gene encoding stearoyl-CoA desaturase, a key regulator of lipid and carbohydrate metabolism. Diabetologia 47:2168-2175

9. Ntambi JM, Miyazaki M, Stoehr JP, Lan H, Kendziorski CM, Yandell BS, Song Y, Cohen P, Friedman JM, Attie AD (2002) Loss of stearoyl-CoA desaturase-1 function protects mice against adiposity. Proc Natl Acad Sci USA 99:11482-11486

10. Kunesova M, Hainer V, Tvrzicka E, Phinney SD, Stich V, Parizkova J, Zak A, Stunkard AJ (2002) Assessment of dietary and genetic factors influencing serum and adipose fatty acid composition in obese female identical twins. Lipids 37:27-32

11. Okada T, Furuhashi N, Kuromori Y, Miyashita M, Iwata F, Harada K (2005) Plasma palmitoleic acid content and obesity in children. Am J Clin Nutr 82:747-750

12. Warensjo E, Ohrvall M, Vessby B (2006) Fatty acid composition and estimated desaturase activities are associated with obesity and lifestyle variables in men and women. Nutr Metab Cardiovasc Dis 16:128-136

13. Michaelsson K, Lithell H, Vessby B, Melhus H (2003) Serum retinol levels and the risk of fracture. N Engl J Med 348:287-294

14. Olofsson H, Byberg L, Mohsen R, Melhus H, Lithell H, Michaelsson K (2005) Smoking and the risk of fracture in older men. J Bone Miner Res 20:1208-1215

15. Boberg M, Croon LB, Gustafsson IB, Vessby B (1985) Platelet fatty acid composition in relation to fatty acid composition in plasma and to serum lipoprotein lipids in healthy subjects with special reference to the linoleic acid pathway. Clin Sci (Lond) 68:581-587

16. Ohrvall M, Berglund L, Salminen I, Lithell H, Aro A, Vessby B (1996) The serum cholesterol ester fatty acid composition but not the serum concentration of alpha tocopherol predicts the development of myocardial infarction in 50-year-old men: 19 years follow-up. Atherosclerosis 127:65-71

17. Warensjo E, Riserus U, Vessby B (2005) Fatty acid composition of serum lipids predicts the development of the metabolic syndrome in men. Diabetologia 48:1999-2005

18. Bandsma RH, Rake JP, Visser G, Neese RA, Hellerstein MK, van Duyvenvoorde W, Princen HM, Stellaard F, Smit GP, Kuipers F (2002) Increased lipogenesis and resistance of lipoproteins to oxidative modification in two patients with glycogen storage disease type 1a. J Pediatr 140:256-260

19. Lee PJ, Patel JS, Fewtrell M, Leonard JV, Bishop NJ (1995) Bone mineralisation in type 1 glycogen storage disease. Eur J Pediatr $154: 483-487$
20. Schwahn B, Rauch F, Wendel U, Schonau E (2002) Low bone mass in glycogen storage disease type 1 is associated with reduced muscle force and poor metabolic control. J Pediatr 141:350-356

21. Rake JP, Visser G, Huismans D, Huitema S, van der Veer E, Piers DA, Smit GP (2003) Bone mineral density in children, adolescents and adults with glycogen storage disease type Ia: a crosssectional and longitudinal study. J Inherit Metab Dis 26:371-384

22. Cabrera-Abreu J, Crabtree NJ, Elias E, Fraser W, Cramb R, Alger $S$ (2004) Bone mineral density and markers of bone turnover in patients with glycogen storage disease types I, III and IX. J Inherit Metab Dis 27:1-9

23. Atteh JO, Leeson S (1983) Effects of dietary fatty acids and calcium levels on performance and mineral metabolism of broiler chickens. Poult Sci 62:2412-2419

24. Atteh JO, Leeson S (1984) Effects of dietary saturated or unsaturated fatty acids and calcium levels on performance and mineral metabolism of broiler chicks. Poult Sci 63:2252-2260

25. Hellerstein MK (1999) De novo lipogenesis in humans: metabolic and regulatory aspects. Eur J Clin Nutr 53(Suppl 1):S53-65

26. Lands WE (1995) Long-term fat intake and biomarkers. Am J Clin Nutr 61:721S-725S

27. Fukuchi S, Hamaguchi K, Seike M, Himeno K, Sakata T, Yoshimatsu H (2004) Role of fatty acid composition in the development of metabolic disorders in sucrose-induced obese rats. Exp Biol Med (Maywood) 229:486-493

28. Corwin RL, Hartman TJ, Maczuga SA, Graubard BI (2006) Dietary saturated fat intake is inversely associated with bone density in humans: analysis of NHANES III. J Nutr 136:159-165

29. Ali AA, Weinstein RS, Stewart SA, Parfitt AM, Manolagas SC, Jilka RL (2005) Rosiglitazone causes bone loss in mice by suppressing osteoblast differentiation and bone formation. Endocrinology 146:1226-1235

30. Botolin S, Faugere MC, Malluche H, Orth M, Meyer R, McCabe LR (2005) Increased bone adiposity and peroxisomal proliferatoractivated receptor-gamma2 expression in type I diabetic mice. Endocrinology 146:3622-3631

31. Schwartz AV, Sellmeyer DE, Vittinghoff E, Palermo L, LeckaCzernik B, Feingold KR, Strotmeyer ES, Resnick HE, Carbone L, Beamer BA, Park SW, Lane NE, Harris TB, Cummings SR (2006) Thiazolidinedione use and bone loss in older diabetic adults. J Clin Endocrinol Metab 91:3349-3354

32. Barish GD, Evans RM (2004) PPARs and LXRs: atherosclerosis goes nuclear. Trends Endocrinol Metab 15:158-165

33. Hamerman D (2005) Osteoporosis and atherosclerosis: biological linkages and the emergence of dual-purpose therapies. QJM 98:467-484

34. Tanko LB, Christiansen C, Cox DA, Geiger MJ, McNabb MA, Cummings SR (2005) Relationship between osteoporosis and cardiovascular disease in postmenopausal women. J Bone Miner Res 20:1912-1920

35. Sundstrom J, Lind L, Vessby B, Andren B, Aro A, Lithell H (2001) Dyslipidemia and an unfavorable fatty acid profile predict left ventricular hypertrophy 20 years later. Circulation 103:836841 